\title{
The performance of RTK-GPS for surveying under challenging environmental conditions
}

\author{
In-Su Lee ${ }^{1}$ and Linlin $\mathrm{Ge}^{2}$ \\ ${ }^{1}$ Cadastral Research Institute, Korea Cadastral Survey Corp., Seoul, Korea \\ ${ }^{2}$ School of Surveying and Spatial Information Systems, University of New South Wales, Sydney, Australia
}

(Received April 5, 2005; Revised December 28, 2005; Accepted January 4, 2006; Online published May 12, 2006)

\begin{abstract}
The aim of this paper was to assess the availability of RTK GPS (Real-Time Kinematic Global Positioning Systems) under some challenging environmental conditions. Testing was performed under two of these conditions, i.e. a small tree-covered garden and a pylon for high-voltage electricity supply. The increase in the number of satellites had many benefits for the integer ambiguity resolution, but no significant contribution to the accuracy of RTK positions and PDOP (Precision Dilution of Position). The results of RTK testing were compared against results from Total Station surveying as a further quality check. The results indicate that integrating RTK GPS system with Total Station is favoured for surveying in urban environments.
\end{abstract}

Key words: RTK-GPS, Total Station surveying, urban environments, garden, pylon.

\section{Introduction}

Global Positioning Systems (GPS) have become more and more important for Earth science (e.g. disaster monitoring), geodesy, land surveying, cadastral surveying and maritime surveying, etc. But unfortunately, on their own, they do not guarantee the centimetre level of accuracy (Martin $e t$ al., 2000).

For scientific applications of GPS, a static GPS is now widely utilized, especially for geophysical phenomena, by measuring the carrier phase of a GPS signal. It is expected that highly accurate static positioning measurements will be useful for detecting the motion of tectonic plates and predicting earthquakes and volcanic processes. GPS can also be used to measure the precipitable water vapour from propagation delay of GPS satellite signals (Yoshihara et al., 2000). Atmospheric sensing is possible through the realtime GPS networks (Ware et al., 2000).

To achieve more accurate coordinates of positions, differential GPS technique is required, which allows even centimetre level accuracy in positioning using the so-called integer ambiguity resolution technique. The basic concept of differential technique is to mitigate the main error sources, such as ionospheric and tropospheric delay, orbit errors and satellite clock errors by receiving satellite signals at a wellknown location. All common errors between base receiver and the rover unit are cancelled out (Landau et al., 2001).

Nowadays, this differential technique is used in real time as well as in post-processing of data. And real-time data transfer is routinely possible, which enables real time computation of baseline vectors (Hofmann-Wellenhof et al., 1997) and has led to the Real-Time Kinematic (RTK) technique.

Copyright (c) The Society of Geomagnetism and Earth, Planetary and Space Sciences (SGEPSS); The Seismological Society of Japan; The Volcanological Society of Japan; The Geodetic Society of Japan; The Japanese Society for Planetary Sciences; TERRAPUB
This paper focuses on assessing the availability of RTK GPS for Earth science under some challenging environmental conditions.

\section{Earth Surveying Methods}

\subsection{RTK GPS}

In the 1980's and early 1990's, results from all GPS surveys were only available after the survey had been completed and the data post-processed. Post-processing provides robust baseline estimates as all measurements can be manipulated a number of times using least squares estimation techniques. The restrictions of post-processing from the surveyors perspective are that field checks and setouts cannot be performed (Timothy and George, 1999).

RTK GPS is the dynamic GPS positioning technique using short observation time; this system provides precise results in real time. To achieve higher positioning accuracies (decimeter or centimeter level) in real time, the double differencing technique should be implemented using carrier phase data.

Carrier phase-based positioning relies on the ability to eliminate, or significantly reduce the common measurement biases across observations made simultaneously by ground receivers to the ensemble of visible GPS satellites. If GPS observations made by a receiver to several satellites are differenced, the receiver dependent biases are eliminated (principally the receiver clock bias).

Furthermore, the pseudo-range measurements are made using the $\mathrm{C} / \mathrm{A}$ code on the $\mathrm{L} 1$ carrier. The same procedure may be applied to carrier phase measurements (which can be made on either the L1 or L2 carrier), to yield in metric units, a similar relation:

$$
\begin{aligned}
\nabla \Delta \Phi_{k i}^{s q}= & \Phi_{k}^{s}-\Phi_{k}^{q}-\Phi_{i}^{s} \\
& -\Phi_{i}^{q}-\lambda \cdot \nabla \Delta N_{k i}^{s q}+\lambda \cdot \nabla \Delta v_{k i}^{s q}
\end{aligned}
$$

Where $\Phi$ is carrier phase measurement, $\lambda$ is the wave- 


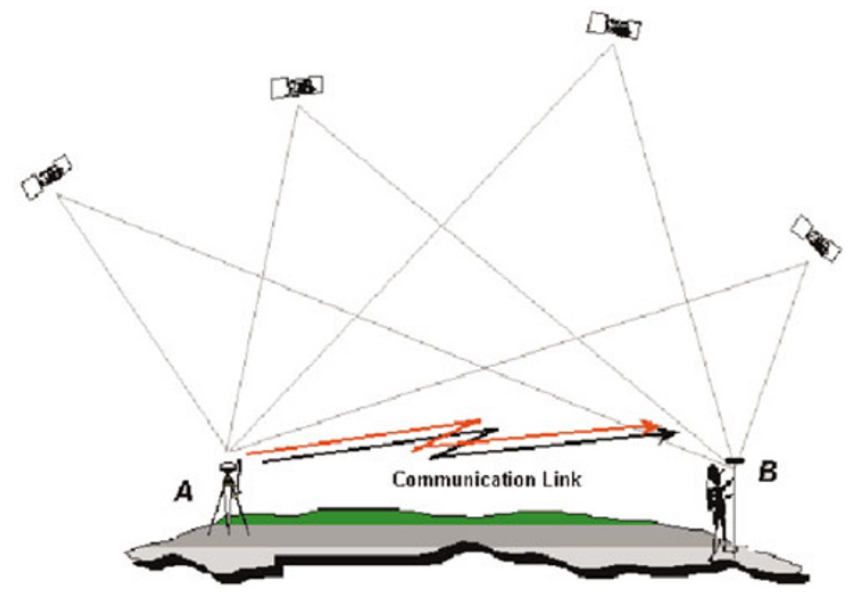

Fig. 1. RTK GPS

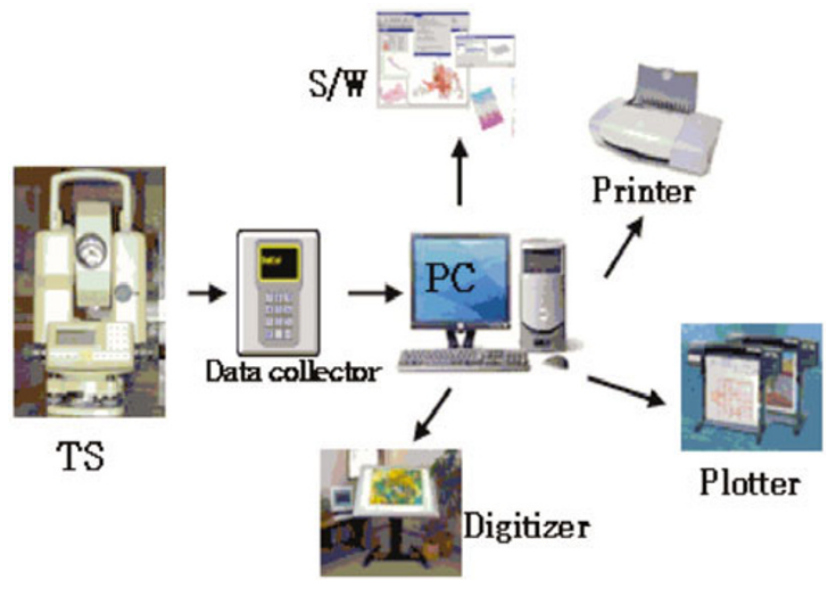

Fig. 2. Application tools of Total Station. length (L1 or L2), $N$ is a constant representing the unknown integer ambiguity, $v^{\prime}$, lower measurement noise. Note that $\nabla \Delta N_{k i}^{s q}$, the double-differenced ambiguity term is an integer. The noise of the double-differenced phase observable is less than one centimeter (Rizos, 1999). The use of double-differenced carrier phase is problematic because any data processing scheme must ensure the estimation of two classes of parameters: (a) the baseline parameters $\Delta x_{k i}, \Delta y_{k i}, \Delta z_{k i}$ (contained within the geometric range term $\Delta \rho_{k i}^{s q}$ ), and (b) the ambiguity parameter $\nabla \Delta N_{k i}^{s q}$ (for each pair of independent satellites s, q). Once the ambiguities have been resolved, all carrier phase data can be converted to a range-like observable, which can be used for precise (centimeter-level) instantaneous positioning.

There are an increasing number of applications for high precision carrier phase based positioning, for machine and vehicle guidance and control. Nowadays kinematic carrier phase based positioning can be carried out in real-time if an appropriate communications link is provided over which the carrier phase data collected at a static base receiver can be made available to the rover receiver's onboard computer to generate the double-differences, resolve the ambiguities and perform the position calculations (Rizos, 1999). This is defined as "Real Time Kinematic" (RTK) GPS positioning. Figure 1 presents a graphical overview of RTK GPS.

\subsection{Total Station surveying}

In the past, a slat and a rope ruler, Electromagnetic Distance Meter (EDM), and Total Station (TS) were used in Earth surveying. But there is a range of shortcomings, e.g., time consuming, laborious and costly, and the need for a direct line of sight. TS is one of the representative and popular equipments in ground surveys. Generally, distance is measured by EDM, and angle, by Transit or Theodolite, but TS can deliver these combined functions. Nowadays, computation and drawing in batch processing could be done with TS. The application tools of TS were shown in Fig. 2.

\section{Case Study 1-At a Garden 3.1 Field observations}

In order to assess RTK GPS under various environmental conditions, a test site was surveyed twice, once in the winter and once in the spring. Herein, RTK1 refers to performance assessment carried out in the winter and RTK2, that in the spring. Figure 3 presents a photograph of a test site taken in the winter and in the spring. In the winter, trees were naked of their leaves and in the spring, full of green foliage.

For this campaign, GPS equipment consisted of a pair of Trimble dual-frequency 4700SSi receivers with firmware allowing RTK GPS observations. The manufacturer's specifications for GPS receivers in horizontal component are $\pm(20 \mathrm{~mm}+2 \mathrm{ppm}$ RMS $)$ (single frequency), $\pm(5 \mathrm{~mm}+1$ ppm RMS) (double frequency) in static mode, and $\pm(20$ $\mathrm{mm}+2$ ppm RMS) in RTK GPS mode when more than 5 satellites are tracked for at least 2 epochs. And to establish a robust wireless data broadcast network for real-time, high-precision GPS, the proper radio modem is required. The optimal and typical ranges of TRIMTALK 450S radio modem utilized in this study are $10 \mathrm{~km}$ and $3-5 \mathrm{~km}$ respectively.

On the other hand, TS, Leica TC-1000 model has the accuracy of the order of 1 second in angle measurement and $\pm(2 \mathrm{~mm}+2 \mathrm{ppm}$ RMS $)$ in distance measurement. TS surveying was carried out to check the performance of RTK GPS.

The area of the test site is $5881.043 \mathrm{~m}^{2}$. The maximum distance between base and the other end of a test site is about $147.643 \mathrm{~m}$. And the number of observables collected through the data collector, TSC1, made by Trimble Navigation Limited, is 178 (RTK1) and 314 (RTK2).

\subsection{Processing and analysis}

This section deals with the accuracy of positions measured, the variation of Position Dilution of Precision (PDOP) with respect to the number of satellite visible. Figure 4 presents the distribution of RTK 1 and RTK 2 measurements.

First, the rate of fixed solution and float solution of (carrier phase) integer ambiguity was investigated to check the quality of RTK measurements. The flags in Fig. 4(b) show that positions have fixed solutions of integer ambiguity. But in Fig. 4(a), it is hard to find a flag. In RTK1, 169 out of 178 points measured, had float solutions of integer ambiguity and the rest of them, fixed solutions (4.47\%). On the other hand, in RTK2, 150 out of 314 points measured, had float solutions of integer ambiguity and the rest of them, 


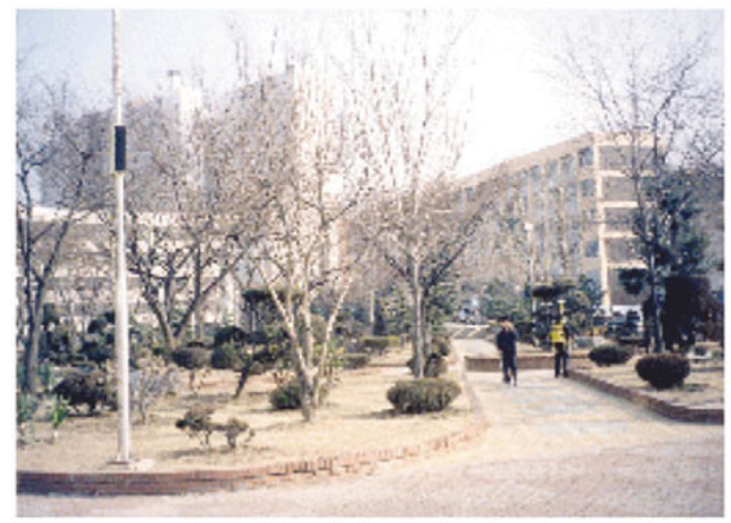

(a)

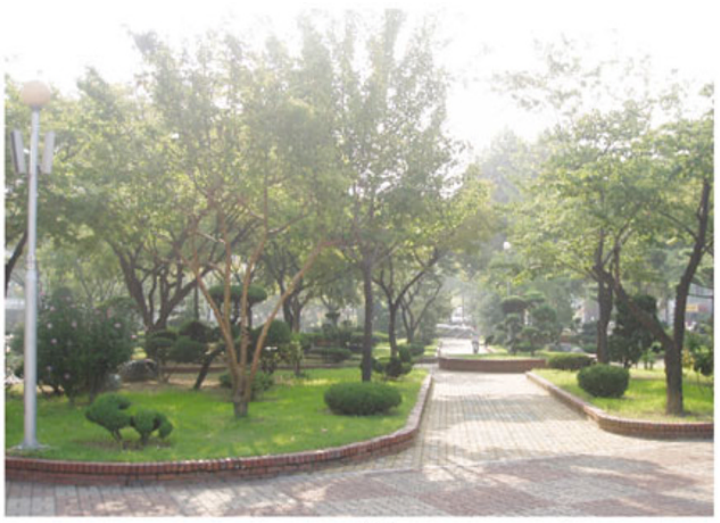

(b)

Fig. 3. Test sites, RTK1 (a) and RTK2 (b).

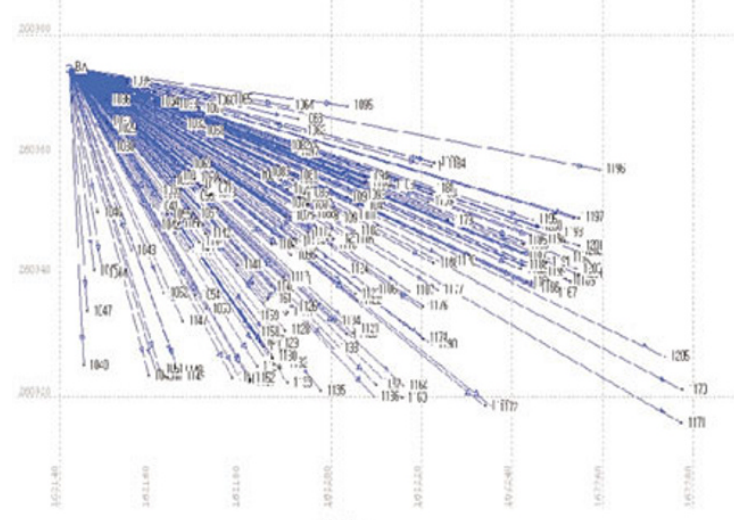

(a)

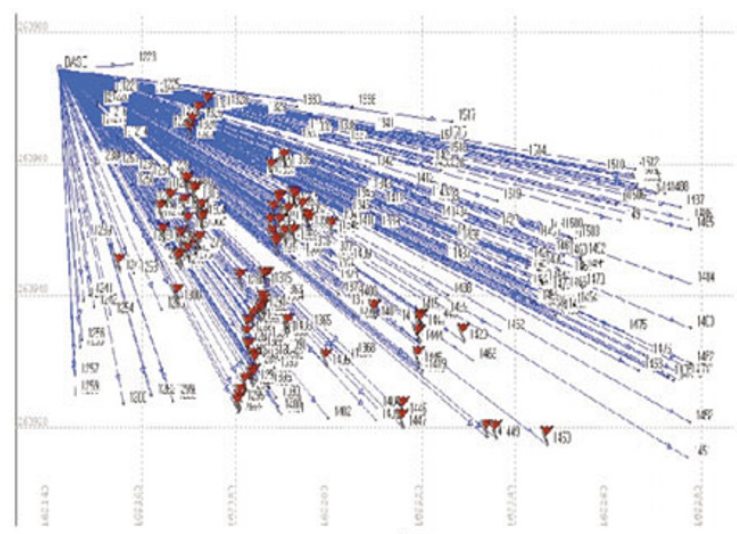

(b)

Fig. 4. Measurements' distribution, RTK1 (a) and RTK2 (b).

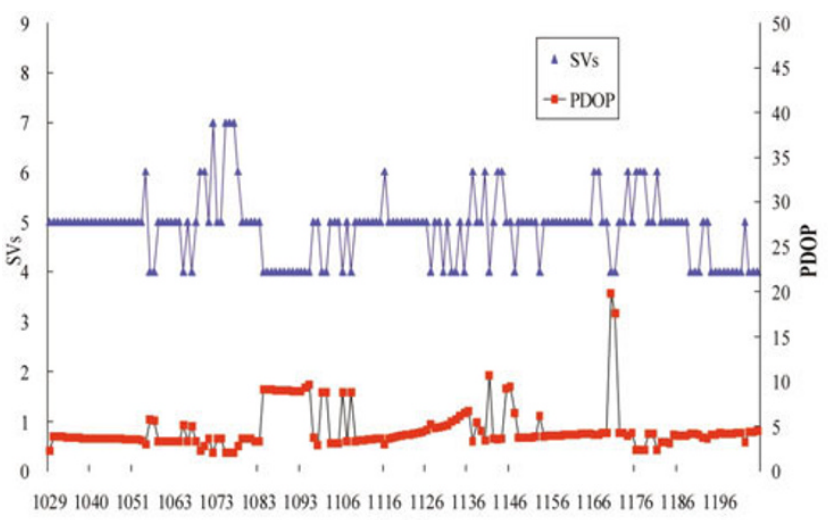

(a)

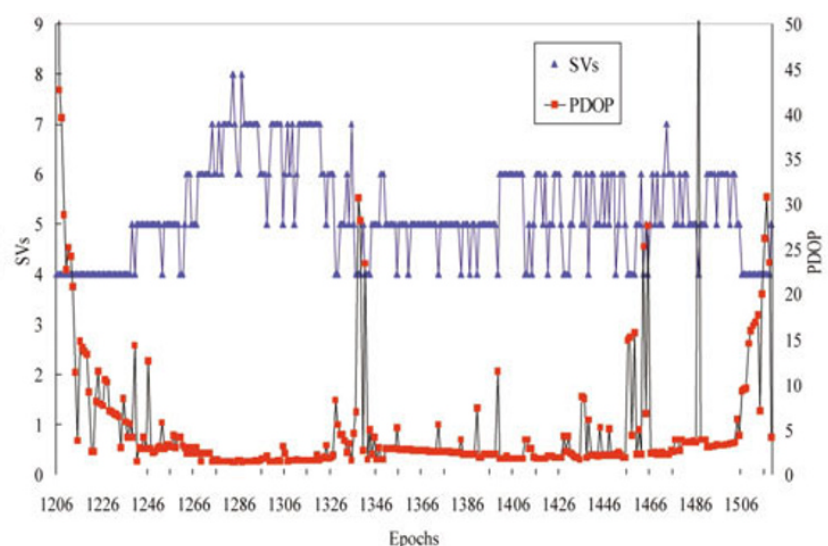

(b)

Fig. 5. PDOP vs. SVs, RTK1 (a) and RTK2 (b).

fixed solutions $(47.77 \%)$.

To obtain the fixed solutions of integer ambiguity, at least 5 satellites must be tracked and the quality of signals from satellites also have to be favourable.

Hence, because a good geometry were maintained in RTK2 during the surveyed time, these results were very likely.

Mean RMS errors in horizontal and vertical components at RTK2 were smaller than those at RTK1, although there were lots of tree leaves in the spring. This may be due to the different number of satellites and the different geometry of GPS satellites being tracked during two field tests. And additionally, all RTK measurements were not analysed in strict conditions because this campaign aimed at only showing the real results of RTK implementation in the urban environments. 


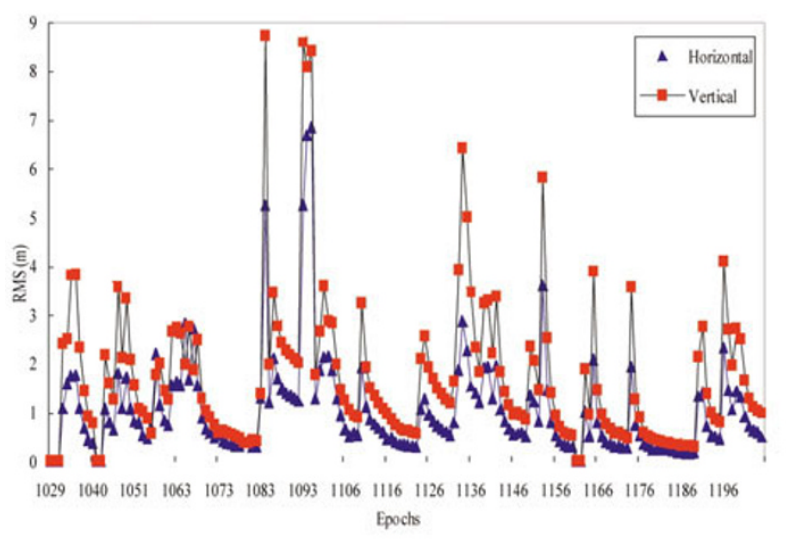

(a)

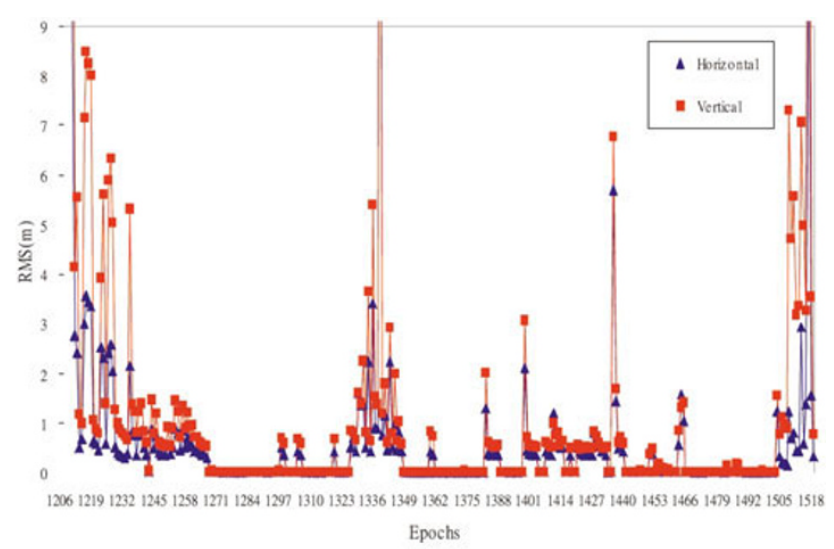

(b)

Fig. 6. RMS vs. Epochs, RTK1 (a) and RTK2 (b).

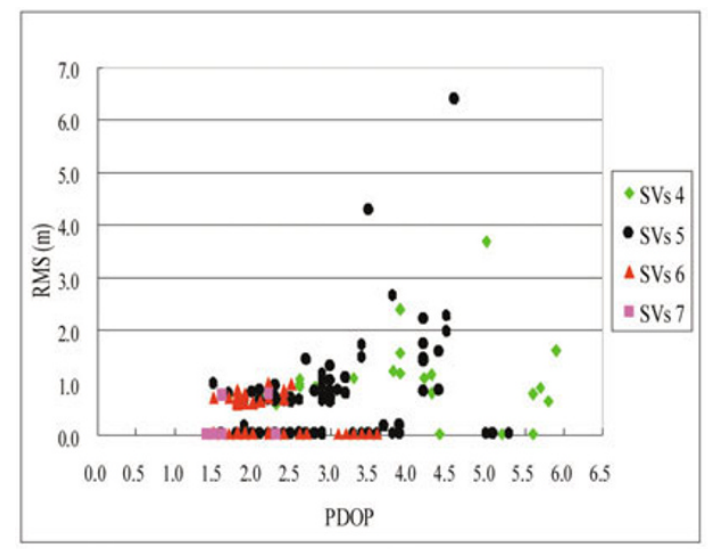

(a)

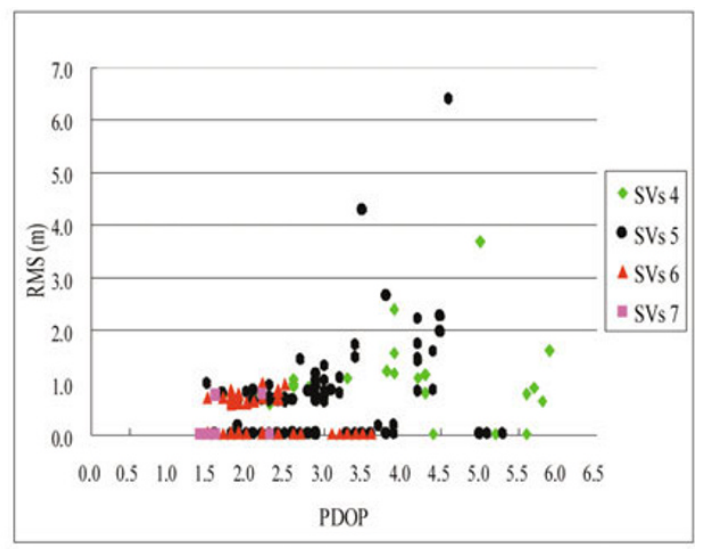

(b)

Fig. 7. RMS, PDOP vs. SVs, RTK1 (a) and RTK 2 (b).

Table 1. Accuracy of points.

\begin{tabular}{ccc}
\hline CASE $\backslash$ ACCURACY & $\begin{array}{c}\text { Horizontal } \\
\text { Mean RMS (m) }\end{array}$ & $\begin{array}{c}\text { Vertical } \\
\text { Mean RMS (m) }\end{array}$ \\
\hline RTK1 & 1.069 & 1.848 \\
RTK2 & 0.786 & 1.490 \\
\hline
\end{tabular}

Secondly, the variation of PDOP with respect to the number of visible satellites tracked was investigated. Dilution of Precision (DOP) is a measure of the quality of the GPS data being received from the satellites and is a mathematical representation for the quality of the GPS position solution. The main factors affecting DOP are the number of satellites tracked and where these satellites are positioned in the sky.

As shown in Fig. 5, the average number of visible satellites in RTK1 and RTK2 was 4.85 and 5.22 and PDOP, 5.2 and 5.41 respectively. Variation of PDOP in RTK2 was rather more turbulent than that in RTK1, although more satellites in RTK2 were tracked (Timothy and George, 1999). Table 1 contains the accuracy of points in horizontal and vertical components.

Figure 6 indicates the RMS in horizontal and vertical components according to epochs. Figure 6(a) showed a big variation (where the RMS of points changes unsteadily) than that of Fig. 6(b), even though trees lose their leaves in the winter. Especially big jumps in RMS were shown at the initial, the middle, and the end part of epochs in RTK2. These were due to GPS rover unit located near and/or under trees with a massive amount of foliage.

Figure 7 (a) and (b) present the RMS, PDOP, versus the number of satellites. These figures show no significant correlation between the RMS and the number of satellites. This is also as is the similar case with PDOP.

In the above, the relation between the number of visible satellites, PDOP and the accuracy were analysed.

At this stage, garden maps of a test site made by TS and RTK system were shown in Fig. 8 and 9. The procedure for these works is as follows:

- Downloading the local Cartesian coordinate of points from each system's storage memory to PC,

- Editing the data using commercially available Computer Aided design (CAD) package, and

- Drawing the garden map.

The garden map obtained from TS was used as ground truth to assess the accuracy of RTK positions in environmental conditions. In Fig. 8, G1-G13 indicate some por- 
Table 2. RMS errors of the local Cartesian coordinates.

\begin{tabular}{cccc}
\hline \multicolumn{4}{c}{ Portion / System RTK1-minus-TS RTK2-minus-TS RTK1-minus-RTK2 } \\
& RMS (m) & RMS (m) & RMS (m) \\
\hline G1 & 0.59 & 0.35 & 0.76 \\
G4 & 1.71 & 0.98 & 1.07 \\
G7 & 1.19 & 2.20 & 2.42 \\
G10 & 5.29 & 4.62 & 2.86 \\
G5 & 0.34 & 0.40 & 0.42 \\
G6 & 1.26 & 0.46 & 1.12 \\
G9 & 1.39 & 1.36 & 1.51 \\
G11 & 3.50 & 3.47 & 1.14 \\
\hline
\end{tabular}

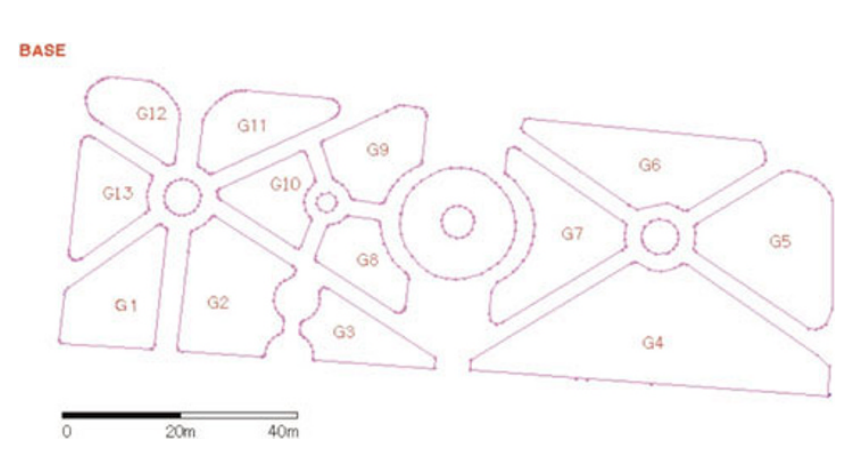

Fig. 8. A garden map by TS.

tions of a garden map derived from TS. The gardens map of Fig. 9(b) looked alike that of Fig. 8, except for some portions.

But, still many mismatches remained on some portions (e.g., G9, G12, and G13) of Fig. 8 and 9(b). Even though many satellites in RTK2 were tracked, in some portions, such as G8 and G10, PDOP was very high and float solution of integer ambiguity often occurred.

Additionally, a radio link was interrupted now and then, even though the base-rover separation was within $100 \mathrm{~m}$. So this could result in Single Point Positioning (SPP) using code only because rover did not obtain any kinds of observables from the base station.

Lastly, three garden maps based on the same coordinate frame were overlapped (Fig. 10) and then, the local Cartesian coordinates of overlapped portions using CAD package were extracted. Local Cartesian coordinates extracted were not included in this paper due to limited space.

Table 2 shows only RMS errors of the difference of local Cartesian coordinates between three systems, that is, RTK1, RTK2, and TS. Mean RMS errors of RTK1-minusTS, RTK2-minus-TS, and RTK1-minus-RTK2 were 2.811 $\mathrm{m}, 2.245 \mathrm{~m}$, and $1.767 \mathrm{~m}$ respectively. These large errors might be caused due to the degradation of the accuracy of positioning by RTK test, with all kinds of factors being mixed together. And large RMS errors of coordinate differences between each system occurred at several portions of the garden map such as G4, G7, G9, G10, and G11.

\section{Case Study 2-At an Electric Power Pylon 4.1 Field Observations}

A test site was selected, considering the possibility of the detection of the factors affecting the accuracy of RTK GPS. Figure 11 shows two electric power pylons. Among them,

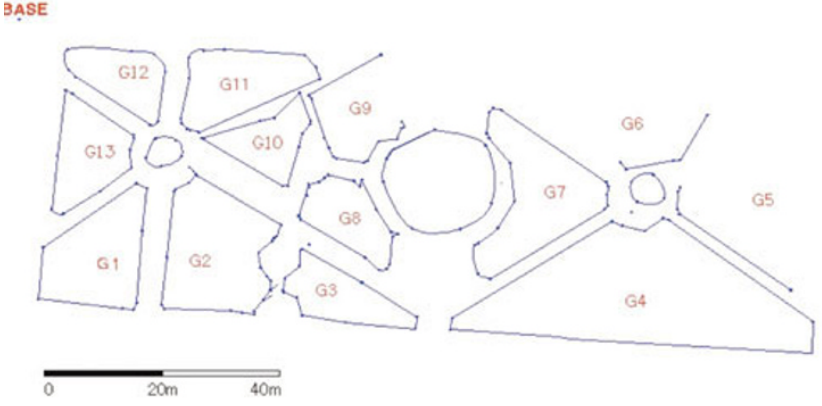

(a)

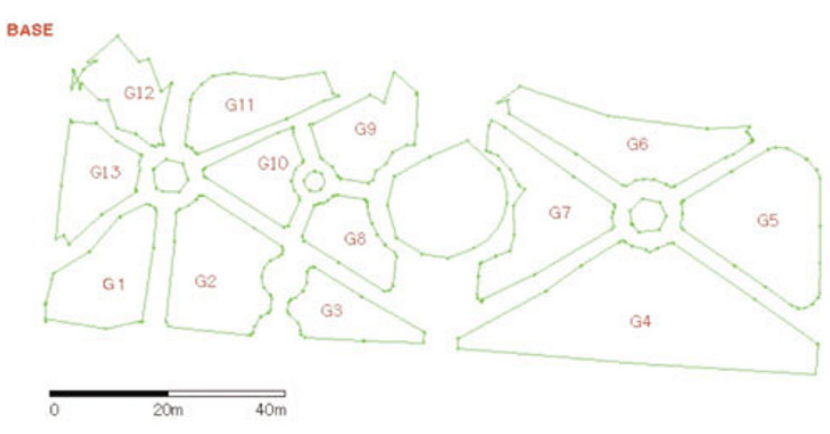

(b)

Fig. 9. Garden maps from RTK1 (a) and RTK2 (b).

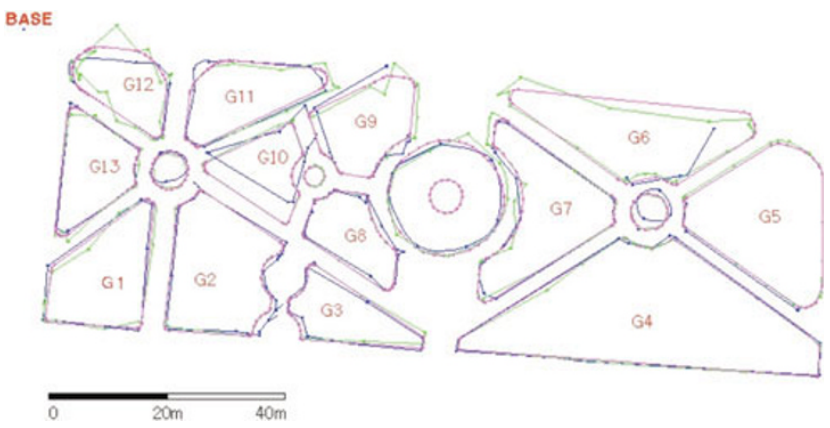

Fig. 10. Overlapped maps from TS, RTK1 and RTK2 surveys.

one has 43 meters of vertical clearance above the ground, $154 \mathrm{kV}$ in voltage, and has no obstructions to blockade tracking the signal of GPS satellites (Fig. 11(a)), and the other, $22 \mathrm{~m}$ in height, the same voltage as previous one, but was surrounded with a few trees (Fig. 11(b)). The distance between two electric pylons is about 150 meters.

These experiments consisted of three categories as followings: (i) static GPS, (ii) RTK GPS, and (iii) TS surveying. And for these campaigns, the same type of GPS and TS system used in Case study1 was used.

In the static GPS, 14 stations were established near the two electric power pylons.

As shown in Fig. 11, 7 stations were established according to various locations and distribution around the electric power pylon. Stations 1 and 11 were approximately 5 meters away from the pylon, stations 2 and 12, between two corner stones of the pylon, station 3, 4, 5, 6 and station 14, $15,16,17$, on the each corner stone of the pylon, and stations 7, 13, the center of four corner stones of the pylon. And the power was turned on during the experiment. 


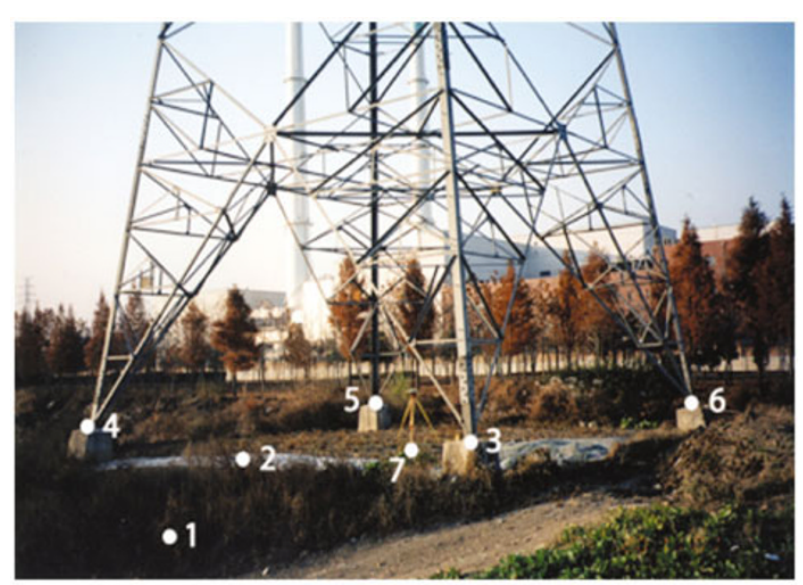

(a)

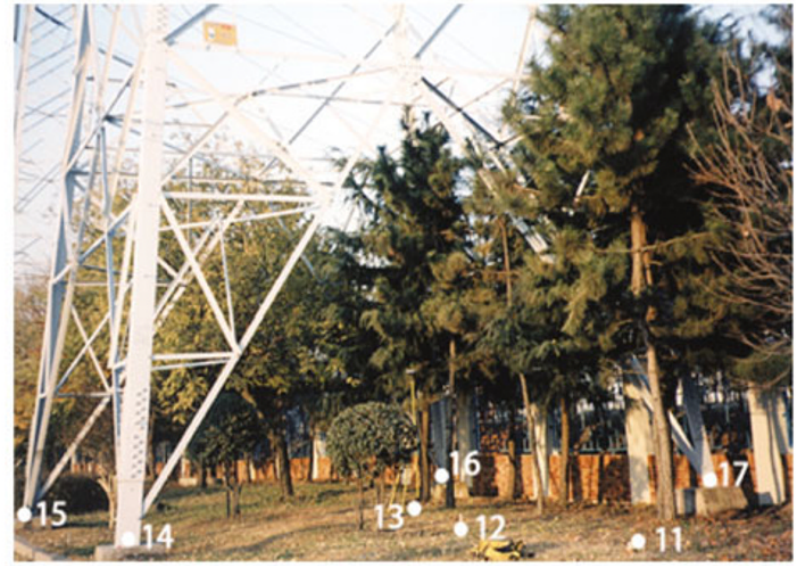

(b)

Fig. 11. Electric power pylons, a static GPS, Pylon -A (a) and Pylon-B (b).

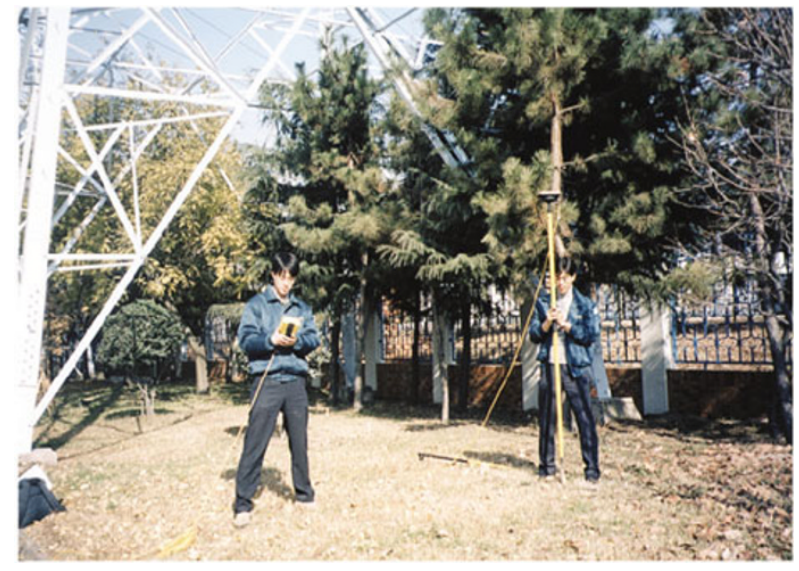

Fig. 12. Electric power pylon, RTK GPS (Pylon-B).
Table 3. RMS errors of adjusted coordinates in WGS-84 coordinate frame.

\begin{tabular}{cccc}
\hline Stations & $\mathrm{sX}(\mathrm{mm})$ & $\mathrm{sY}(\mathrm{mm})$ & $\mathrm{sZ}(\mathrm{mm})$ \\
\hline 1 & 107.1 & 85.8 & 71.7 \\
2 & 138.8 & 144.4 & 183.0 \\
3 & 147.3 & 140.7 & 150.1 \\
4 & 286.2 & 191.6 & 137.8 \\
5 & 157.5 & 225.6 & 120.0 \\
6 & 198.3 & 199.4 & 123.3 \\
7 & 148.4 & 148.1 & 135.6 \\
11 & 138.0 & 147.5 & 172.3 \\
12 & & & \\
13 & 1060.7 & 584.8 & 444.6 \\
14 & 189.3 & 265.3 & 152.2 \\
15 & 188.0 & 251.7 & 127.2 \\
16 & 351.6 & 441.1 & 350.0 \\
17 & & & \\
\hline
\end{tabular}

conducted by occupying the other stations around the pylon in sequence. Generally, it took 10 to 30 -seconds per one station to obtain the fixed or floating solution of carrier phase integer ambiguity.

Figure 12 shows the overview of RTK GPS implementation on spot. Currently, GPS antenna was located at station, about $5 \mathrm{~m}$ away from one of pillars of pylon in Fig. 11(b).

Table 3 contains RMS errors of adjusted coordinates of stations in WGS-84 coordinate frame. In Table 3, station 1 to 7 had best accuracy of decimeter level in $\mathrm{X}, \mathrm{Y}$ and $\mathrm{Z}$ components in WGS-84 coordinate frame, but not so, in stations 11 to 17 . Especially, stations 12 and 17 were not adjusted in the network, leading to no values in Table 3. This might be attributed to the interference of satellite signal due to tree canopy and the electric power pylon itself.

Table 4 contains the local Cartesian coordinates obtained from RTK, a static GPS, and TS surveying and Table 5 shows the coordinate differences in horizontal components between RTK, a static GPS, and TS surveying.

RTK-minus-TS had the average coordinate differences of $-0.046 \mathrm{~m},-0.052 \mathrm{~m}$, and static GPS-minus-TS, $-0.395 \mathrm{~m}$, $0.141 \mathrm{~m}$, and static GPS-minus-TS, $0.253 \mathrm{~m},-0.153 \mathrm{~m}$ in horizontal components, respectively. Figure 14 illustrates a in a static GPS and TS surveying was utilized as the base station throughout the surveyed time. Observations were 


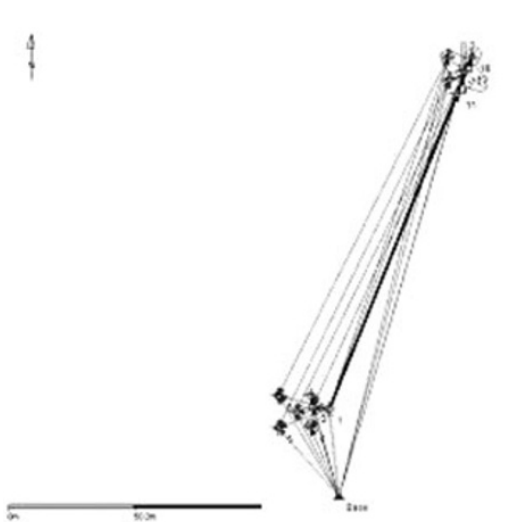

(a)

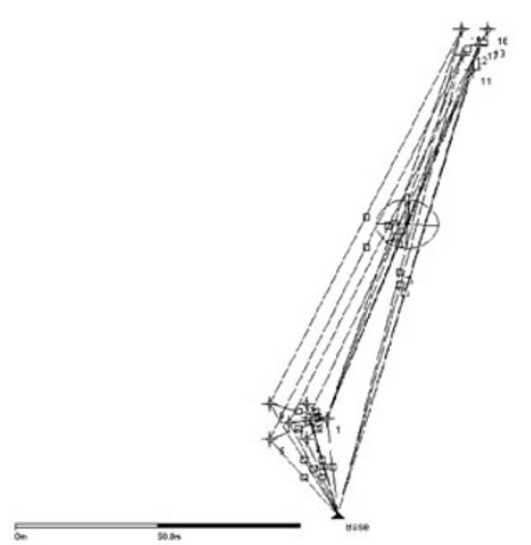

(b)

Fig. 13. Baseline processing (a) and network adjustment (b).

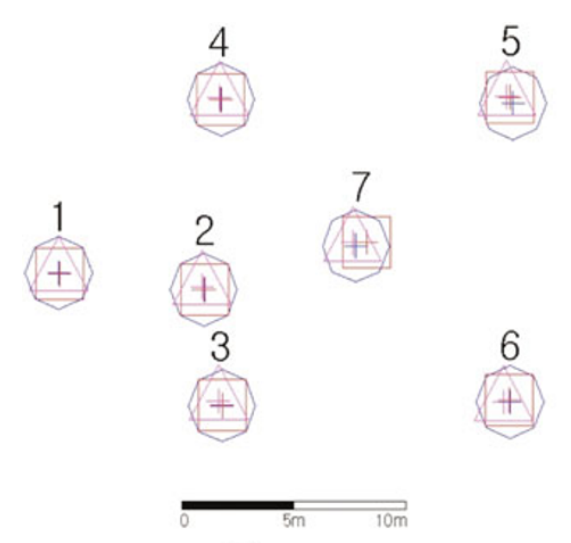

(a)

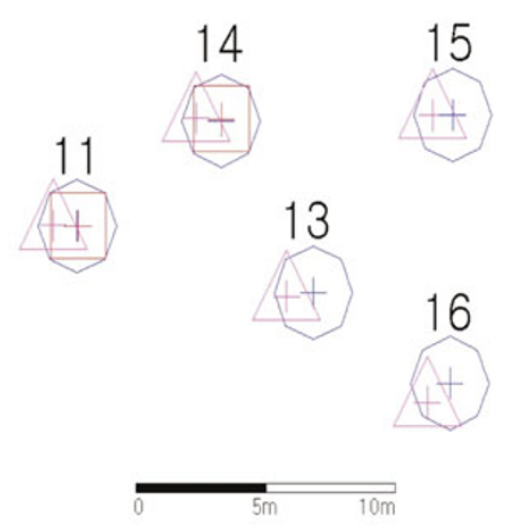

(b)

Fig. 14. Location diagram from RTK $(\triangle)$, Static GPS $(\bigcirc)$, and TS ( $\square$ ) for Pylon-A (a) and Pylon-B (b).

Table 4. Comparison of local Cartesian coordinates.

\begin{tabular}{ccccccc}
\hline Stations & \multicolumn{2}{c}{ RTK } & \multicolumn{2}{c}{ TS } & \multicolumn{2}{c}{ Static } \\
& $\mathrm{X}(\mathrm{m})$ & $\mathrm{Y}(\mathrm{m})$ & $\mathrm{X}(\mathrm{m})$ & $\mathrm{Y}(\mathrm{m})$ & $\mathrm{X}(\mathrm{m})$ & $\mathrm{Y}(\mathrm{m})$ \\
\hline 1 & 258963.053 & 154028.031 & 258962.997 & 154028.082 & 258962.997 & 154028.082 \\
2 & 258963.673 & 154021.575 & 258963.695 & 154021.625 & 258963.555 & 154021.683 \\
3 & 258968.470 & 154020.805 & 258968.489 & 154020.806 & 258968.307 & 154020.968 \\
4 & 258955.840 & 154020.853 & 258955.821 & 154020.852 & 258955.728 & 154020.927 \\
5 & 258955.727 & 154007.954 & 258955.981 & 154007.811 & 258955.706 & 154008.090 \\
6 & 258968.249 & 154007.970 & 258968.332 & 154007.938 & 258968.350 & 154008.222 \\
7 & 258961.728 & 154014.351 & 258961.883 & 154014.817 & 258961.754 & 154014.974 \\
11 & 259089.108 & 154079.889 & 259089.077 & 154079.900 & 259088.151 & 154079.882 \\
12 & & & & & & \\
13 & & & 259098.158 & 154082.045 & 259097.132 & 154082.168 \\
14 & 259094.623 & 154076.443 & 259094.609 & 154076.511 & 259093.641 & 154076.424 \\
15 & & & 259103.517 & 154076.321 & 259102.739 & 154076.333 \\
16 & & & 259103.412 & 154084.951 & 259102.544 & 154085.577 \\
17 & & & & & \\
\hline
\end{tabular}

location diagram of the stations computed from RTK $(\Delta)$, a static GPS $(\bigcirc)$, and TS ( $\square$ ). In Pylon-A, RTK, a static GPS and TS surveying showed the similar coordinates of positions at each station 1-7 (Table 4).

But at stations 11-16 around Pylon-B, RTK was con- ducted only at station 11 and 14, and a static GPS, only at station $11,13,14,15$ and 16 , thereby some of squares ( $\square$ ) and triangles $(\triangle)$ were not shown in Fig. 13(b). Static GPS is conducted with the collection of sufficient epochs than RTK GPS, thereby leading to resolve integer ambiguity of 
Table 5. Comparison of local Cartesian coordinates' difference.

\begin{tabular}{ccccccc}
\hline Stations & \multicolumn{2}{c}{ RTK - minus - TS } & \multicolumn{2}{c}{ Static - minus - TS } & \multicolumn{2}{c}{ RTK - minus - Static } \\
& DX $(\mathrm{m})$ & DY $(\mathrm{m})$ & DX $(\mathrm{m})$ & DY $(\mathrm{m})$ & DX $(\mathrm{m})$ & DY $(\mathrm{m})$ \\
\hline 1 & 0.06 & -0.05 & 0.00 & 0.00 & 0.06 & -0.05 \\
2 & -0.02 & -0.05 & -0.14 & 0.06 & 0.12 & -0.11 \\
3 & -0.02 & -0.00 & -0.18 & 0.16 & 0.16 & -0.16 \\
4 & 0.02 & 0.00 & -0.09 & 0.08 & 0.11 & -0.07 \\
5 & -0.25 & 0.14 & -0.28 & 0.28 & 0.02 & -0.14 \\
6 & -0.08 & 0.03 & 0.02 & 0.28 & -0.10 & -0.25 \\
7 & -0.16 & -0.47 & -0.13 & 0.16 & -0.03 & -0.62 \\
11 & 0.03 & -0.01 & -0.93 & -0.02 & 0.96 & 0.01 \\
12 & & & & & & \\
13 & & & -1.03 & 0.12 & & \\
14 & 0.01 & -0.07 & -0.97 & -0.09 & 0.98 & 0.02 \\
15 & & & -0.78 & 0.01 & & \\
16 & & & -0.87 & 0.63 & & \\
17 & & & & & & \\
\hline
\end{tabular}

carrier phase more accurately and stably.

Large differences between Static GPS and TS surveying occurred at stations 15 and 16 . These could be attributed to the lack of the number of satellites being tracked at that moment and the interference of satellite signal due to tree canopy and electric power pylon itself (Alsalman, 2001).

\section{Conclusions}

This paper dealt with the performance assessment of RTK GPS at small garden under the urban environments, in two different seasons, and underneath the electric power pylon surrounded by tree canopy. In addition, Total Station surveying as ground truth was conducted at the same time.

In Case study 1, the analysis of result showed that many satellites were tracked during the surveyed time, although a test site was full of a mass of green foliage in the spring, except for under the tree canopy and/or very close to buildings. And it was proven that the number of satellites tracked had no significant influence on the position accuracy and PDOP.

In Case study 2, electric power pylon surveying was successfully performed in Static GPS, but not so good in RTK GPS. But, in open area, RTK GPS is expected to be very useful tool for electric power pylon surveying.

The two case studies highlighted the weakness of RTK GPS due to e.g. the blockage of satellite signals near the buildings and/or under the tree canopy. Therefore, the usage of RTK GPS will be dependent on the applications, the error tolerance of the applications, and the size of fields.
Acknowledgments. This work was supported by the Postdoctoral Fellowship Program of Korea Science \& Engineering Foundation (KOSEF).

\section{References}

Alsalman, A. S. A., Effects of electric power lines on the accuracy of GPS positioning, Survey Review, 36, 279, 54-61, 2001.

Hofmann-Wellenhof, B., H. Lichtenegger, and J. Collins, GPS: Theory and Practice, pp. 100-138, Springer-Verlag, Wien New York, 1997.

Landau, H., U. Vollath, A. Deking, and C. Palels, Virtual reference Station Networks - Recent Innovations by Trimble, Technical Paper, Trimble Terrasat GmbH, November, pp. 1-14, 2001.

Martin, S. and C.-H. Jahn, Experiences in set-up and usage of a geodetic real-time differential correction network, LETTER, Earth Planet Space, 52, 851-855, 2000.

Rizos, C., GPS enhancements, in Notes on Basics GPS Positioning and Geodetic Concepts, edited by C. Rizos, School of Surveying and Spatial Information, University of New South Wales, pp. 15-16, electronic version, 1999.

Timothy, R. L. and P. G. George, The Influence of the Number of Satellites on the Accuracy of RTK GPS Positions, THE AUSTRALIAN SURVEYOR, Vol. No. 1, 64-70, 1999.

Ware, R. H., D. W. Fulker, S. A. Stein, D. N. Anderson, S. K. Avery, R. D. Clark, K. K. Droegemeier, J. P. Kuettner, J. Minster, and S. Soroosh, Real-time national GPS networks: Opportunities for tmospheric sensing, Earth Planets Space, 52, 901-905, 2000.

Yoshihara, T., T. Tsuda, and K. Hirahara., High time resolution measurements of precipitable water vapor from propagation delay of GPS satellite signals, Earth Planets Space, 52, 479-493, 2000.

I.-S. Lee (e-mail: lis9919@yahoo.co.kr) and L.Ge (e-mail: 1.ge@unsw. edu.au) 